\title{
Energy Optimal Path Algorithm for routing in Static Adhoc Network using Greedy Approach (EOGR)
}

\author{
Anshu Chaturvedi \\ D. N. Goswami \\ Tripti Sharma \\ Deptt. of Computer Application SOS in computer Science and Applications Research Scholar \\ M.I.T.S., Gwalior \\ Jiwaji University, Gwalior \\ jiwaji University, Gwalior
}

\begin{abstract}
Wireless adhoc networks are excellent area for researchers with an algorithm background. In this paper we have given an algorithmic approach to the problem of routing with minimum energy consumption by the ad hoc network. we have proposed an energy optimal path algorithm used for routing in static adhoc networks using greedy approach of algorithm design. We have given the mathematical proof of correctness of proposed algorithm. We also performed simulation to show the effectiveness \& correctness of our algorithm. Simulation results show that the proposed algorithm perform well in comparison to the GPSR algorithm in terms of energy, throughput $\&$ other factors.
\end{abstract}

Keywords : Adhoc Network ,Greedy method, Energy consumption, Optimization

\section{INTRODUCTION}

In recent years the widespread availability of wireless communication and handheld devices has stimulated research on self-organizing networks that do not require a preestablished infrastructure. These ad hoc networks, as they are commonly called, consist of autonomous nodes that collaborate in order to transport information. The issues in adhoc networks always bring the attention of research community. The fundamental issues of connectivity, scalability, energy consumption, routing and topology control in ad hoc networks is worth to study. Graph theory plays an important role in the study of these fundamental issues. Many research problem turn out be variants of classical graph theory problems. Every node of a wireless ad hoc network is a user terminal and a router at the same time. The management of the network is distributed between all nodes. Therefore, it is extremely necessary to have efficient routing algorithms which make it possible to exchange data over paths consisting of multiple nodes.Ad hoc networks can be subdivided into two classes: static and mobile. In static ad hoc networks the position of a node may not change once it has become part of the network. Typical examples are rooftop networks [1].In mobile ad hoc networks, nodes may move arbitrarily. Examples where mobile ad hoc networks may be used are the establishment of connectivity among handheld devices or between vehicles. For the remainder of this work we will solely focus on static ad hoc networks.

This is verified from the facts[2,3] that geographical routing protocols are more scalable than topology based routing protocols like Dynamic Source Routing(DSR),Destination Sequence Distance Vector(DSDV), and Ad hoc On Demand Distance Vector(AODV) routing protocols[4]. In geographical routing algorithms, routing decisions are based on local decisions. Therefore they do not require bulky routing tables to maintain routing as in link state [5] or distance vector routing schemes. [6].

Communication \& computation capabilities of the wireless ad hoc networks are limited by the battery power of the network nodes, which are limited energy resources. Therefore, more emphasis is being given to develop various techniques to reduce energy consumption of the wireless adhoc network. To reduce the energy consumption or to make the optimal use of the energy in the adhoc network various attempts are made in which routing decision is modified by including energy factor to it. We have proposed an energy optimal path algorithm for static wireless ad hoc network, which will provide a complete routing path for forwarding the packets from source to destination taking moderate consideration of energy as well as distance. The routing decision will be taken at each node beginning with the source $\&$ will depend upon not only on the distance from destination but also on some energy consumption criteria defined for the nodes. Since at each step of routing decision, packet is forwarded to the node which has minimum cost in terms of energy \& distance from the destination, therefore the path given by this algorithm is energy optimal as the total energy consumption of the whole routing path(from source to destination) is minimum. As we mentioned earlier that we are going to use algorithmic approach therefore we will have a look at various algorithm design techniques $\&$ which one will suit to our problem. There are various algorithm design techniques which we can use to write the algorithm for this energy optimal path problem like Greedy Method, Dynamic Programming, Branch $\&$ Bound Method etc. Since in our problem routing decision is taken locally, we have taken greedy method for designing the proposed algorithm .A greedy algorithm finds the optimal solution of the problem by taking local optimal decisions. A greedy algorithm assumes that a local optimum is a part of a global optimum. Moreover this method is easy to apply \& gives us best solution in few steps. In some cases, the solution given by greedy method sometimes may not be optimal but it is closest to the optimal. And we are always interested in optimal or nearer to optimal solution, so this method is a straightforward way to obtain the best solution with in the given constraints.

Since we are using the concept of Graph Theory, We have modeled static ad hoc network as a weighted graph. We applied our algorithm to the modeled graph \& apply the concepts of graph theory to prove the correctness of our algorithm. We have also shown that the proposed algorithm is based on the greedy approach by showing that the proposed algorithm exhibits the properties of greedy algorithm. We have given it a name as EOGR.

The paper is organized as follows. Section 2 presents the related work. Section 3 describes the proposed work. Section 4 describes the methodology of proposed algorithm. Section 5 presents the algorithm followed by verification of algorithm in section 6. Section 7 discusses simulation \& performance evaluation. Finally, section 8 presents conclusion \& future work. 


\section{RELATED WORK}

This section describes the overview of the previous related work.

\subsection{Greedy Routing:}

In greedy routing, when a node sends a packet to a particular destination, the destination node ID as well as its geographical position is included in the packet header. If the destination is not directly reachable, then the packet is forwarded to the one of the neighboring node, which has minimum distance to the destination. Such a procedure of forwarding the packet to the neighboring node is repeated at the intermediate nodes also. In case a forwarding node could not find a neighbor node that lies closest to the destination than itself, then greedy routing fails. This situation is known as a hole in the geographical distribution of the nodes.

Karp and Kung proposed make it possible to recover from this hole by introducing GPSR [7], a geographic routing protocol that uses a planer subgraph of the wireless network graph to route through the holes. GPSR uses the greedy routing as soon as it recovers from holes or dead-ends.

SWING \{ Small World Iterative Navigation Greedy) Routing Protocol in MANETs finds shorter routes than geometric routing protocol though with a longer route establishment time. More important thing about the SWING is that, SWING works even if position information is inaccurate. A theoretical proof is also given in this paper[8]. In Greedy MFR (Most Forward Within Radius)[9], source node or packet forwarder node, sends packet to one of its neighbors with most forward progress towards destination node (closest neighbor to destination). Greedy MFR uses combination of metrics distance-velocity similarity-power, to decide about giving the packet to which neighbor as only using distance deciding metric in Greedy to forward packet to a neighbor node is not suitable for all conditions.

\subsection{Energy Efficient Routing:}

Because of power limiting factor of wireless adhoc networks, presently many researchers are trying to develop a routing protocol which reduces the energy consumption during the communication and increase the life of the nodes which in turn increases the connectivity of the network. Here is a short description of the protocols which have already been proposed with the aim to reduce energy consumption of the node. Power aware routing [10] is one such kind of protocol in which routing path determination depends on the use of different energy aware metrics. The Localized Energy Aware Routing (LEAR) protocol [11] which is mainly based on DSR protocol, has been proposed to control the energy consumption. In this protocol, each node keeps the information of its remaining power level so that it can decide whether or not to accept the route request message to find the routing path. When the remaining power level of the node is higher than a threshold value the route request message is accepted otherwise it is dropped.

In span protocol [12], most of the nodes enter into a power saving sleep mode without much reducing the capacity or connectivity of the networks. Modified AODV protocol is proposed by adding expected residual life time of node in basic AODV protocol. This modified version is Energy consumption speed-based routing [13] .The multi-cost routing protocol [14], as the name indicates consists of several cost parameters associated with each link. This multi-cost uses three metrics of the path: (1) number of hops, (2) minimum remaining energy left on nodes and (3) maximum or sum of transmission powers of path nodes. In EAGR [15] that is proposed for sensor networks, packet is forwarded to the neighbor who is having energy level above than threshold and has distance equal to or less than the average distance of all its neighbors and among those neighbors having maximum energy level. In GEAR (Geographic and Energy Aware Routing)[16] for wireless sensor networks the entire ad hoc network is divided in to grids. If an intermediate node lies within the same grid as that of the destination node, the packet is flooded to the entire grid; otherwise, the packet is forwarded to the best next hop with in the largest weighted cost (of the distance to the destination and the energy available).Energy Aware Greedy Routing Scheme [17] for wireless adhoc networks used multi-cost routing protocol in which packet is forwarded to the node which has minimum cost. This multi-cost uses three metrics of the path: (1) rate of energy consumption (2) fraction of energy consumed (3) distance from destination.

An algorithmic Approach to Geographic routing in Ad hoc and Sensor Networks [18] presents a specific routing algorithm based on a synthesis of the greedy forwarding and face routing approaches, and provides an algorithm analysis of the presented work.

We have also given an algorithmic approach to our work \& used greedy method to write the algorithm for the discussed problem. In the previous work done so far regarding the problem discussed in this paper, only simulation results were given to show the correctness of their proposed work but in our work besides the simulation, we present the mathematical proof for the verification of our algorithm. This provides a concrete argument for the correctness of our algorithm.

\section{PROPOSED WORK}

Routes in the greedy routing protocol are based on a single metric i.e. minimum distance from the destination. It has a drawback that usually only one path for each sourcedestination pair is selected. This consideration of single metric might have a bad effect when nodes in a communication network have limited energy. The nodes which are repetitively participating in the routing will discharge soon because of their limited energy. Moreover, this can also have a bad effect on network connectivity, because some of the nodes will be dead faster than others.

Concerning to the above problem, we propose an Energy Optimal Path Algorithm for routing in static ad hoc networks. Here, we will be able to determine a routing path which forwards the packet with least energy consumption as well as with least distance.

\section{METHODOLOGY OF THE PROPOSED WORK}

In proposed work, we considered static ad hoc network, means the nodes in the network are static.so that we can model ad-hoc network as a graph. The wireless ad hoc network is modeled by a connected graph $G=(V, E)$, where $\mathrm{V}$ is the set of wireless hosts (hereinafter referred as nodes) and $\mathrm{E}$ represents set of wireless links between pair of nodes. We make following assumptions for the ad hoc networks considered in our study.

- All nodes are homogeneous, i.e. maximum wireless transmission ranges are identical and all the receiver nodes have same signal detection threshold level while receiving the signal.

- Nodes are located on a two dimensional plane A link $(\mathrm{u}, \mathrm{v})$ exists between nodes $\mathrm{u}$ and $\mathrm{v}$ only if the 
nodes are located within each others wireless transmission ranges. Hence, the resulting graph is undirected.

- All such nodes, which are located within the maximum wireless transmission range of a node $u$ are known as its one-hop neighbors. In this paper, we will refer them as neighbors only.

Now we elaborate various parameters used in the proposed scheme.

\subsection{Amount of Energy Consumption}

Amount of energy consumption is defined as the total energy consumption per unit initial energy of the node. We have assumed that $A_{\text {in }}$ is the Amount of energy consumed for nth periodic interval up to the start of $(n+1)$ th periodic interval.

$$
A_{\text {in }}=E_{\text {io }}-E_{\text {in }} / E_{\text {io }}
$$

where $E_{\text {in }}$ be the energy at the start of the nth periodic interval and $E_{i o}$ be the initial energy of the node $i$. We did not consider the rate of energy consumption because we considered the network to be static \& nodes to be homogenous so rate of energy consumption is going to remain constant $\&$ it will not going to have much effect on cost computation parameter.

\subsection{Cost Computation Parameter}

When a source node wants to send the packet to the destination node, and the distance of source node to destination node is $\mathrm{D}$ and Distance of its neighbors to destination is $\mathrm{d}_{1}, \mathrm{~d}_{2}, \mathrm{~d}_{3}, \ldots . \mathrm{d}_{\mathrm{k}}$, then we can get the moderate consideration of energy at nodes if the packet is forwarded to the node, which has least amount of energy consumption $\left(\mathrm{A}_{\text {in }}\right)$. .Furthermore, moderate consideration of distance traveled can also be achieved if the packet is forwarded to the node which is at the minimum distance from the destination.

The value of $A_{\text {in }}$ is always less than or equal to 1 and for $d_{i}<$ $\mathrm{D}$, the value of $\mathrm{d}_{\mathrm{i}} / \mathrm{D}\left(\mathrm{W}_{\mathrm{i}}\right.$ or distance metric) is less than 1 . Thus for the above condition, we can add amount $\mathrm{A}_{\text {in }}$ and distance metric $\mathrm{W}_{\mathrm{i}}$ for the equal consideration of energy consumed \& distance traveled.

So that total cost of the routing path will be optimal with respect to energy \& distance. We propose the cost metric as

$$
C_{\text {in }}=\left(W_{i}+A_{\text {in }}\right) \text {, where } W_{i} \text { is equal to } d_{i} / D
$$

We designed the algorithm on the basis of greedy method,[19]in which we try to be greedy on this cost metric .We know that in case of shortest path problem Greedy method always yields the optimal solution. That's why we apply this method so that we can obtain lowest energy path. Total energy of the path will be minimum as we always choose the node which has least amount of energy consumed.

\section{PROPOSED ALGORITHM}

As mentioned earlier we have modeled ad hoc network as an undirected connected graph, now we can formulate the problem as-

Consider a weighted connected graph $\mathrm{G}=(\mathrm{V}, \mathrm{E})$ with cost function $\mathrm{C}: \mathrm{E}->\mathrm{R}$ mapping edge to real valued cost. Cost incurred when we travel from one node to other through the edges. The cost of the path $P=\left\langle v_{0}, v_{1}, \ldots ., v_{k}\right\rangle$ is the sum of the costs of its constituent edges.

$$
\mathrm{C}(\mathrm{P})=\sum \mathrm{C}\left(\mathrm{v}_{\mathrm{i}-1}, \mathrm{v}_{\mathrm{i}}\right)
$$

$$
\mathrm{i}=1 \text { to } \mathrm{k}
$$

we define the optimal path cost from $\mathrm{u}$ to $\mathrm{v}$ by

$\delta(\mathrm{u}, \mathrm{v})=$

$\min \{\mathrm{C}(\mathrm{P}): \mathrm{u} \rightarrow \mathrm{v}\}$, if there is a path from $\mathrm{u}$ to $\mathrm{v}$

\} or

NULL , otherwise
Here the cost metric associated with a node is defined as above in the previous section, i.e. the cost metric in going from vertex $\mathrm{u}$ to $\mathrm{v}$ is given as:

$C[v]=\left(W_{v}+A_{v}\right)$ where $W_{v}$ is equal to $d_{v} / D$

We are interested in optimizing the total cost of the path.

\subsection{Algorithm:}

Input to the algorithm: A Graph $\mathrm{G}=(\mathrm{V}, \mathrm{E})$, where $\mathrm{V}$ is the set of the vertices $\& E$ is the set of the edges . Source node $=\mathrm{s} \&$

Destination node $=\mathrm{f}$

Output of the algorithm: A set $\mathrm{R}$ of vertices which will represent an energy optimal path; if greedy forwarding is successful. \& NULL otherwise

The Algorithm repeatedly selects the vertex u $\varepsilon \mathrm{V}$-s with minimum cost estimate ,adds $\mathrm{u}$ to $\mathrm{R} \&$ checks all the edges leaving $\mathrm{u}$ means its neighbors. In the following implementation, we use a minimum priority queue $\mathrm{Q}$ of vertices keyed by their e values. [where e is the estimation cost of that node from source.]A variable Flag associated with each vertex indicates whether that vertex has been checked to be taken in to consideration for the optimal path.

BEGIN:

// Calculate the distance of source to destination

$D=\sqrt{ }\left(X_{s}-X_{f}\right)^{2}+\left(Y_{s}-Y_{f}\right)^{2}$

// Calculate the distance of every vertex except the source, to the destination

For every vertex $v \& \quad V-\{s\}$

$$
d_{v}=\sqrt{ }\left(X_{v}-X_{D}\right)^{2}+\left(Y_{v}-Y_{D}\right)^{2}
$$

For every vertex $v \varepsilon V-\{s\}$

$$
W_{v}=d_{v} / D
$$

// Calculate the cost metric of every vertex except the source

For every vertex $v \& V-\{s\}$

$C[v]=\left(W_{v}+A_{v}\right)$

For each vertex $v \& V[G]$

$$
\begin{aligned}
\text { Do } & \\
e[v] & \leftarrow \infty \\
e[s] & \leftarrow 0 \\
\text { Flag }[v] & \leftarrow \text { false } \\
R & \leftarrow \Phi \\
Q & \leftarrow V[G]
\end{aligned}
$$

While $Q \neq \Phi$

I

Do

\{

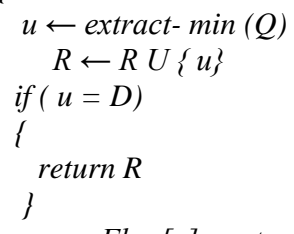

Flag $[u] \leftarrow$ true
For each vertex $v \&$ Adj $[u] \&$ Flag $[v] \leftarrow$ false $\& d_{v}<D$ do

$$
\text { i }
$$$$
\text { if } e[v]>e[u]+C[v]
$$$$
\text { then } e[v] \leftarrow e[u]+C[v]
$$

\}

if $R \neq \Phi$

then return $R$

else

return NULL

Finish 


\section{VERIFICATION OF THE ALGORITHM:}

Before giving the mathematical verification of the algorithm, we would like to describe some facts:

\subsection{Optimal Substructure of Optimal Path:}

Optimal path algorithm typically rely on the property that an optimal path between two vertices contains other optimal paths within it. This optimal sub-structure property is a hallmark of the applicability of greedy method [20].

The following lemmas state the optimal sub-structure property of optimal path more precisely.

\section{Lemma 6.1. 1: (Sub optimal paths of optimal paths are optimal)}

Given a weighted graph $\mathrm{G}=(\mathrm{V}, \mathrm{E})$ with cost function $\mathrm{C}$ : $\mathrm{E}$ $\rightarrow \mathrm{R}$ (here $\mathrm{R}$ is a real number). Let $\mathrm{P}=<$ $\mathrm{v}_{1}, \mathrm{v}_{2}, \ldots \ldots \ldots \ldots \ldots \mathrm{v}_{\mathrm{k}}>$ be the optimal path from vertex $\mathrm{v}_{1}$ to $\mathrm{v}_{\mathrm{k}}$ and for any $\mathrm{i} \& \mathrm{j}$ such that $1 \leq \mathrm{i} \leq \mathrm{j} \leq \mathrm{k}$, let $\mathrm{P}_{\mathrm{ij}}=\left\langle\mathrm{v}_{\mathrm{i}}\right.$, $v_{i+1}, \ldots \ldots \ldots v_{j}>$ be the sub path of $P$ from vertex $v_{i}$ to vertex $v_{j}$. Then $\mathrm{P}_{\mathrm{ij}}$ is a optimal path from $\mathrm{v}_{\mathrm{i}}$ to $\mathrm{v}_{\mathrm{j}}$.

Proof: If we decompose the path $P$ in to $v_{1} \rightarrow v_{i} \rightarrow v_{j} \rightarrow v_{k}$, then we have that $\mathrm{C}(\mathrm{P})=\mathrm{C}\left(\mathrm{P}_{1 \mathrm{i}}\right)+\mathrm{C}\left(\mathrm{P}_{\mathrm{ij}}\right)+\mathrm{C}\left(\mathrm{P}_{\mathrm{jk}}\right)$.

Now assume that there is a path $\mathrm{P}{ }_{\mathrm{ij}}$ from vi to vj with $\operatorname{cost} \mathrm{C}\left(\mathrm{P}^{\prime}{ }_{\mathrm{ij}}\right)<\mathrm{C}\left(\mathrm{P}_{\mathrm{ij}}\right)$.Then $\mathrm{v}_{1} \rightarrow \mathrm{v}_{\mathrm{i}} \rightarrow \mathrm{v}_{\mathrm{j}} \rightarrow \mathrm{v}_{\mathrm{k}}$ is a path from $\mathrm{v}_{1}$ to $\mathrm{v}_{\mathrm{k}}$ whose cost $\mathrm{C}\left(\mathrm{P}_{1 \mathrm{i}}\right)+\mathrm{C}\left(\mathrm{P}_{\mathrm{ij}}\right)+\mathrm{C}\left(\mathrm{P}_{\mathrm{jk}}\right)$. $<\mathrm{C}(\mathrm{P})$, Which contradicts the assumption that $\mathrm{P}$ is a optimal path from $\mathrm{v}_{1}$ to $\mathrm{v}_{\mathrm{k}}$.

Now we can give the proof of correctness of our algorithm: Let $\mathrm{e}[\mathrm{v}]$ be the estimation cost label found by the algorithm for vertex $\mathrm{v} \&$ let $\delta[\mathrm{s}, \mathrm{v}]$ be the optimal path from $\mathrm{s}$ to $\mathrm{v}$. We want to show that $\mathrm{e}[\mathrm{v}]=\delta[\mathrm{s}, \mathrm{v}]$ for every vertex $\mathrm{v}$ at the end of the algorithm, showing that the algorithm correctly computer the path. We prove this by induction on $|R|$ via the following lemma. [where $\mathrm{R}$ is the set of vertices forming the optimal path]

\section{Lemma 6.1.2: for each $\mathrm{x} \varepsilon \mathrm{R}, \mathrm{e}[\mathrm{x}]=\delta[\mathrm{s}, \mathrm{x}]$ Proof:}

Base Case $(|R|=1)$ : Since $R$ only grows in size, The only time $|\mathrm{R}|=1$ is when $\mathrm{R}=\{\mathrm{s}\}$ and $\mathrm{e}[\mathrm{s}]=\delta[\mathrm{s}, \mathrm{s}]$. Which is correct.

Inductive Hypothesis: when $|\mathrm{R}| \leq \mathrm{k}$, for each $\mathrm{x} \varepsilon \mathrm{R}, \mathrm{e}[\mathrm{x}]=$ $\delta[\mathrm{s}, \mathrm{x}]$

Let $R$ be the set of size $k$ to which we add $u$. Let R' $=R \quad U$ $\{u\}$. Since $|R|=k$, the lemma holds by the inductive hypothesis for R. We need to show that lemma holds for every vertex in R'. By the inductive hypothesis for every vertex in R' that isn't $\mathrm{u}$, we have the correct estimation cost label. We need only to show that $\mathrm{e}[\mathrm{u}]=\delta[\mathrm{s}, \mathrm{u}]$ to complete the claim.

Let $\mathrm{P}$ be the s- to- $\mathrm{u}$ path that has estimation cost e[u].This path is composed of a path $\mathrm{P}^{\prime}$ from $\mathrm{s}$ to $\mathrm{v} \&$ the edge vu. Suppose there is a optimal path $\mathrm{Q}$ from s- to - u i.e. $\delta[\mathrm{s}, \mathrm{u}]$. Let $\mathrm{y}$ be the first vertex in $\mathrm{Q}$ that is outside $\mathrm{R}$. There must be an edge $x y$ on this path such that $\mathrm{x} \varepsilon \mathrm{R}$. We can break $\mathrm{Q}$ up into the pieces $\mathrm{Q}_{1}$, xy, $\mathrm{Q}_{2}$, where $\mathrm{Q}_{1}$ is the s-to-x subpath of $\mathrm{Q} \&$ $\mathrm{Q}_{2}$ is the y-to-u subpath of $\mathrm{Q}$. now we have

$\mathrm{e}[\mathrm{x}]=\delta[\mathrm{s}, \mathrm{x}]$, By inductive hypothesis,

$\mathrm{e}[\mathrm{y}] \leq \mathrm{e}[\mathrm{x}]+\mathrm{C}[\mathrm{y}]$, By update step of the algorithm,

and by greedy choice property of the algorithm, $\mathrm{e}[\mathrm{u}] \leq \mathrm{e}[\mathrm{y}]$ which in turn must be at most the cost of Q, contradicting that $\mathrm{Q}$ is a optimal path. Therefore $\mathrm{e}[\mathrm{u}]=\delta[\mathrm{s}, \mathrm{u}]$.

This proof shows that the algorithm is correct by applying it for $\mathrm{R}=\mathrm{V}$ [set of the vertices]

\section{SIMULATION \& PERFORMANCE EVALUATION \\ 7.1 Simulation parameters description}

Our proposed scheme was simulated \& evaluated using ns-2 network simulator(version ns-2.34)[21]. As the proposed algorithm is for static wireless adhoc networks therefore the simulation environment used is for static wireless adhoc networks. The network dimension used is $500 * 500 \mathrm{~m}$ square network. The transmission range of each node is assumed to be $100 \mathrm{~m}$. The number of nodes is set to 100 . The position of nodes are chosen at random. The nodes are assumed to be static. The Media Access Control (MAC) layer model used is 802.11 model. Since our aim is to obtain an energy optimal path therefore energy model is used in simulation, in which small amount of energy is given to the nodes of network scenarios i.e. 2.0 joules. Traffic Sources are constant bit rate(CBR) with UDP applications. We consider 5 CBR connections to forward the packet from source to destination. The timing of these CBR connections ranges between 10 to 200 seconds. Data packets are 128 bytes in size and the packets sending rate is initially set to .33 seconds which is varied up to 2.97 seconds.

We measure the following performance metrics for simulation conditions listed above.

a. Packet Delivery Fraction: It is measured as the fraction of generated packets that are received at the destination. We can also define it as the ratio of the number of packets delivered to destination and the number of packets sent by source.

b. Average Throughput: It is measured as the bit rate or byte rate at the destination.

c. Percentage of Energy consumed : It is measured as the ratio percentage of total energy

consumed per unit received packets.

d. Average Latency: It is measured as average delay a packet takes to travel from the source to destination. In other words it is average delay of one packet to reach at destination.

\subsection{Performance Evaluation}

Since our proposed algorithm is based on greedy algorithm therefore we evaluate the performance of our algorithm by comparing it with original GPSR protocol as it is also based on greedy routing. Also in the implementation of our algorithm we take the help of Ke Liu's ns code of GPSR that used ns version ns-2.29[22].

Simulation results observed for various metrics are as follows:

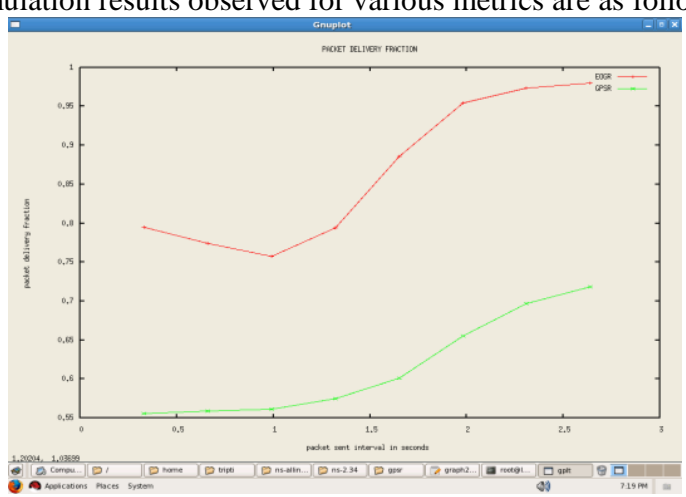

Fig. 1

Figure 1 shows that there is a considerable increase in packet delivery fraction in EOGR as compared to GPSR. This increase is from $34.1 \%$ to $42.02 \%$. 


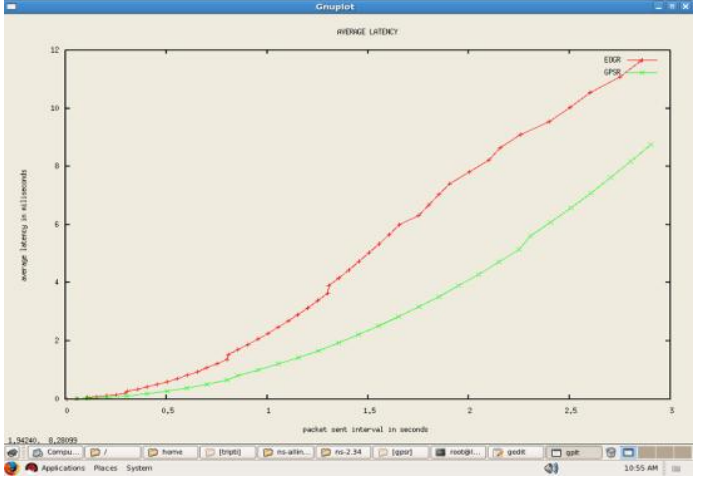

Fig. 2

Fig. 2 shows the comparison of average latency for EOGR \& GPSR. The figure shows that the average latency is increased in case of EOGR as compared to GPSR. This is because GPSR selects the next node only on the basis of shortest distance from the destination. Therefore it is obvious that latency delay in case of GPSR will be less in comparison to EOGR as in EOGR main emphasis is on energy. It selects the next node to forward the packet which has less amount of energy consumption. So the distance travelled may be more.

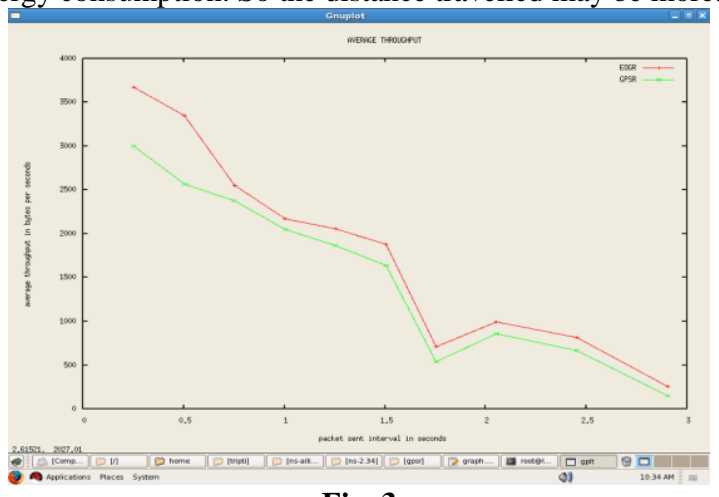

Fig. 3

From the fig. 3 we can see that there is improvement in the throughput in EOGR as compared to GPSR. We know that the node with high rate of energy consumption can not trusted for packet forwarding, as more number of nodes depends on it for routing the packets. It means that node is very important for network connectivity. If we choose that node for forwarding the packet its energy will be depleted \& it will be dead, resulting in network disconnection. Our algorithm (EOGR) prevents this condition by not forwarding the packet to the nodes which has higher rate of energy consumption as compared to all other nodes, thereby improves the network connectivity. Therefore more packet will be delivered to the destination. It is verified from the figure 1 also which shows that the packet delivery fraction is higher in EOGR. More the packet delivered to the destination more will be the throughput. It is obvious from the figure 3.There is a percentage of increase in throughput by EOGR than GPSR varies from $13.6 \%$ to $23.3 \%$.

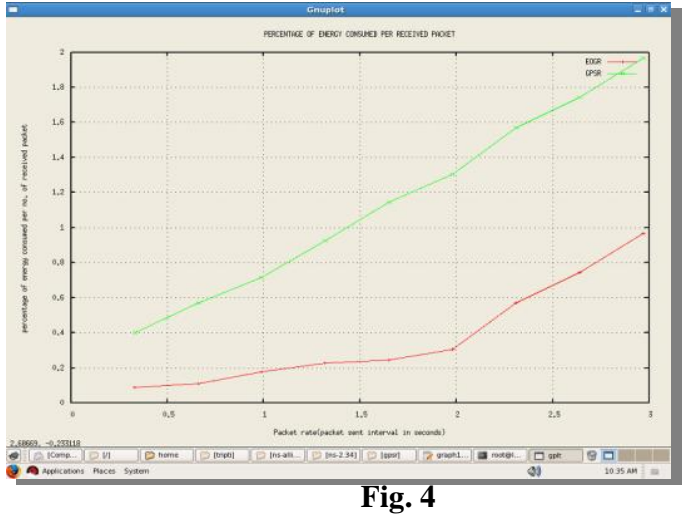

Fig. 4 evinces the energy efficacy of proposed algorithm. We measure energy efficiency by calculating percentage of energy consumption per unit number of packet received. From the fig. 4 it is clear that the energy consumed per unit received packet is always less than the GPSR. GPSR selects \& uses the same path (shortest distance from destination) for the, or until the energy of any node on the path is completely exhausted. As a result, only a small portion of total number of nodes will participate in routing again and again. But in EOGR, amount of energy consumption change over the time, so path selected may not be the same for all the time.

\section{CONCLUSION}

We have proposed an energy optimal path algorithm, which can be used for routing in static wireless ad hoc network. As we have shown above, that this algorithm will result in a path which will be optimal in terms of energy as well as the distance. It will be an energy optimized shortest path or route. We have also shown the correctness of the algorithm. Through this algorithm we have discovered a path which forwards the packet with more reliability, less energy consumption, balanced delay, and balanced overhead and increased throughput. So the connectivity \& life time of network can be improved. This is also verified through simulation. We have given the mathematical proof of correctness of algorithm which is another way of the verification of algorithm. Besides this, we have proved optimal sub-structure property of the algorithm \& the algorithm also possesses greedy choice property. So that we can say that given algorithm is based on greedy algorithm and gives us energy optimal path. We can call our algorithm as an adaptive algorithm as it always adapts with the changing values of energy \& distance $\&$ gives us optimal solution with in given constraints. In future we are trying to modify and implement it for mobile adhoc network also.

\section{REFERENCES}

[1.] D. Beyer, M. D. Vestrich, and J. J. Garcia-Luna-Aceves, The Rooftop Community Network: Free, High-Speed Network Access for Communities, Hurley and Keller, Edrs., The First 100: New Options for Internet and Broadband Access, MIT Press, 1999, pp. 75-91.

[2.] R. Jain, A. Puri and R. Sengupta, Geographical Routing Using Partial Information for Wireless Ad hoc Networks, In IEEE Personal Communication, pp. 48-57 (2001).

[3] J. Li, J. Janotti, D.S.J.De Couto, D.R. Karger, and R. Morris, Scalable Location Service for Geographic Ad hoc Routing, In Proceedings ofIEEE/ACM, MOBICOM, pp. 120-130 (2000). 
[4] E. Royer and C. K. Toh, Review of Current Routing Protocols for Mobile Ad hoc Networks, In IEEE Personal Communication, pp. 85-97 (1999).

[5] T.H.Clausen, G. Hansen, L. Cristensen and G. Behermann, The Optimized Link State Routing Protocol Evaluation through Experiments and Simulations, In IEEE Personal Communication (2001).

[6] C. Perkins, and P. Bhagwat, Highly Dynamic Destination Sequenced Distance Vector Routing (DSDV) for Mobile Computers, In Proceedings of ACM SIGCOMM, pp. 234-244 (1994).

[7] B. Karp and H. T. Kung, GPSR: Greedy Perimeter Stateless Routing for Wireless Networks, In Proceedings of IEEE/ACM MOBICOM, pp.243-254 (2000).

[8] C. Liu and J. Wu, SWING: Small World Iterative Navigation Greedy.

[9] Mahboobeh Abdoos, Karim Faez, and Masoud Sabaei: Position Based Routing Protocol with More Reliability in Mobile Ad Hoc Network in World Academy of Science, Engineering and Technology 492009

[10] S. Singh, M. Woo, C. Raghvendra, Power Aware Routing in Mobile Ad hoc Networks, In Proceedings of IEEE/ACM MOBICOM, pp. 181-190 (1998).

[11] K. Woo, C. Yu, H.Y. Youn, and B. Lee, Non-blocking Localized Routing Algorithm for Balanced Energy Consumption in Mobile Ad hoc Networks, In Proceedings of MASCOTS, pp. 117-124 (2001).

[12] B. Chen, K. Jamieson, H. Balakrishnan and R. Morris, Span: An Energy-Efficient Coordination Algorithm for Topology Maintenance in Ad hoc Networks, In ACM Wireless Networks Journal, Vol. 8, No.5, pp. 481-494 (2002).
[13] L. Romdhani and C. Bonnet, Energy Consumption Speed-Based Routing for Mobile Ad hoc Networks, In Proceedings of ICDCSW,pp. 729-734 (2004).

[14] P.C Kokkinos, C.A. Papageorgiou and E.A. Varvarigos, Energy Aware Routing in Wireless Ad hoc Networks, In Proceedings of WoWMoM,pp. 306-311 (2005).

[15] R. Haider, M. Y. Javed, N. S. Khattak, EAGR: Energy Aware Greedy Routing in Sensor Networks, In Proceedings of Future Generation Communication and Networking, Vol. 2, 344-349 (2007).

[16] Y.Yu, R.Govindan, D. Estrin: Geographical \& Energy Aware Routing: a recursive data dissemination protocol for wireless sensor network.

[17] S. Sharma, H.M. Gupta, S. Dharmaraja: Energy Aware Greedy Routing Scheme for Wireless Ad hoc Networks in SPECTS 2008

[18] F.Kuhn, R.Wattenhofer, A. Zollinger: An algorithmic Approach to Geographic routing in Ad hoc and Sensor Networks in the Networking, IEEE/ACM , volume 16, pp 51-62

[19] E. Horowitz, S. Sahni and S. Rajasekaran,:Fundamental of Computer Algorithm II edition pp 210-222.

[20] T.H. Cormen, C.E. Leiserson, R.L. Rivest, C. Stein :Introduction to Algorithm II edition , pp 581-582.

[21] K.fall, K. Vardhan, NS notes and Documentation, the VINT Project, UC Barkeley, LBL, USC/ISI and Xerox PARC (2000),http://www.isi.edu/nsnam/ns/nsdocumentation.html.

[22] GPSR Code Help: http://www.cs.binghamton.edu/ //kliu/research/ns2code/index.html. 